\title{
Migración y desarrollo en Cuba: socialismo, subdesarrollo productivo y globalización neoliberal
}

\author{
Edel José Fresneda Camacho \\ Raúl Delgado Wise
}

Resumen. Se analiza la especificidad de la migración cubana en los marcos del debate contemporáneo sobre migración y desarrollo. Plantea un marco teórico-conceptual que busca explicar la naturaleza de los ingentes flujos poblacionales que distinguen a Cuba. Argumenta que las contradicciones derivadas del subdesarrollo productivo socialista propician una serie de distorsiones estructurales que restringen las posibilidades de desarrollo humano y derivan en crecientes limitaciones al consumo y a la movilidad social de la mayoría de la población cubana. La migración opera como un mecanismo que compensa parcialmente estas distorsiones a través de dos modalidades: la migración económica y la exportación de servicios intensivos. Ambas modalidades son producto y resultado del modo de producción no capitalista vigente en la isla caribeña.

Palabras clave: migración cubana, subdesarrollo productivo socialista, limitación relativa del consumo, movilidad social, exportación de servicios intensivos.

\begin{abstract}
Aвstract. This article analyzes the specificity of Cuban migration in the context of contemporary debates on migration and development. It provides a theoretical framework that seeks to explain the nature of the vast population flows that characterize Cuba. It argues that the contradictions arising from the socialist productive underdevelopment lead to a series of structural distortions that restrict the possibilities for human development and result in increasing limitations to consumption and social mobility for the majority of the Cuban population. Migration operates as a mechanism to partially offset these distortions through two modalities: economic migration and the export of intensive services. Both modalities are a product and result of the noncapitalist mode of production in force on the Caribbean island.

KEYWORDs: cuban migration, socialist productive underdevelopment, relative limitation to consumption, social mobility, export of intensive services.
\end{abstract}




\section{INTRODUCCIÓN}

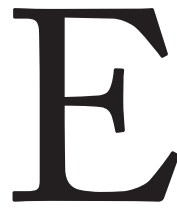

n el contexto de la globalización neoliberal, la migración se inscribe como parte de una embestida global en contra de la clase trabajadora orquestada por las grandes corporaciones multinacionales (cMns), en el marco de una estrategia de dominación imperialista comandada por Estados Unidos (Harvey, 2004). Entre las piezas clave del nuevo engranaje capitalista, hoy en crisis, despuntan: a) el establecimiento de redes globales de capital monopolista, a través de las cuales las grandes corporaciones desplazan partes de sus procesos productivos, comerciales y de servicios hacia la periferia en busca de fuerza de trabajo barata y recursos naturales; b) la reestructuración de los sistemas de innovación, que posibilita a las grandes CMNs poner a su servicio, a través de mecanismos de subcontratación (outsourcing y offshore outsourcing), fuerza de trabajo calificada del Sur, reduciendo costos, transfiriendo riesgos y responsabilidades y beneficiándose de la adquisición de patentes; c) la financiarización que, a través de operaciones especulativas y el recurso al expediente del capital ficticio, favorece las dinámicas de concentración y centralización del capital, y d) la intensificación de la degradación ambiental propiciada por la mercantilización a ultranza de la naturaleza y la privatización de los recursos naturales a escala planetaria, en atención al afán insaciable de conquista y lucro de los poderes imperiales (Márquez y Delgado Wise, 2011).

Para desentrañar la relación entre migración y desarrollo que toma lugar en este contexto, es menester tener presente que con el ingreso de los antiguos países del bloque socialista ${ }^{1}$ y China al mercado capitalista mundial,

${ }^{11 /}$ Aun cuando en este trabajo no se profundiza acerca del llamado socialismo real, es pertinente acotar que en estas formaciones sociales no se siguieron a cabalidad los principios básicos que debieran regir a una sociedad socialista: «a) la propiedad co-

156 MIGRACIÓN Y DESARROLLO, VOL. 11, NÚM. 20, PRIMER SEMESTRE DE 2013 
aunado a la liberación de fuerza de trabajo en la periferia, como consecuencia de la implantación de las políticas de ajuste estructural, la magnitud del ejército laboral de reserva alcanza dimensiones sin precedentes: alrededor de $60 \%$ de la fuerza de trabajo global (Foster et al., 2011). En similar tenor, de acuerdo con datos de la Organización Internacional del Trabajo (отт, 2011): 1530 millones de trabajadores (poco más de la mitad de la fuerza de trabajo mundial) laboran en condiciones de inseguridad, 900 millones reciben un salario de dos dólares al día o menos, al tiempo que 204 millones se ubican en las filas del desempleo. Ello, a su vez, genera incesantes presiones para emigrar en condiciones de vulnerabilidad extrema.

El tipo de movilidad poblacional que predomina bajo la globalización neoliberal es la migración forzada (categoría acuñada por Marx para referirse a la migración irlandesa). Este tipo de migración alude fundamentalmente a desplazamientos Sur-Norte y Sur-Sur, y se asocia a un incremento sin parangón de las asimetrías entre países y regiones, así como a un acrecentamiento de las desigualdades sociales. En este sentido, la migración adquiere una connotación negativa en un doble sentido: por un lado, se imbrica dialécticamente con un aumento y profundización de las brechas entre desarrollo y subdesarrollo capitalistas y, por el otro, guarda relación con situaciones de severa exclusión social, superexplotación laboral, racismo, xenofobia y criminalización a la que son sometidos los trabajadores migrantes (Delgado Wise, 2013).

Los rasgos aludidos, que caracterizan la relación entre migración y desarrollo bajo la globalización neoliberal, no aplican cabalmente al caso de

mún, social, sobre los medios de producción; b) la remuneración de los productores conforme al trabajo aportado a la sociedad; c) la supervivencia del Estado a la vez que se inicia, desde el Estado mismo, el proceso de su propia destrucción; d) la apertura de un espacio cada vez más amplio a la democracia al transformar radicalmente el principio de representatividad, y e) la autogestión social al devolverse a la sociedad las funciones que usurpaba el Estado» (Sánchez Vázquez, 1999). 
la migración en la isla cubana. Aun cuando es ampliamente reconocido que el tema de Cuba reviste singular importancia y creciente significación en el concierto de la migración no sólo latinoamericana sino mundial (tanto por su magnitud relativa como por sus especificidades), lo cierto es que se trata de un fenómeno poco estudiado, sobre todo en lo concerniente a sus particularidades y contrastes en relación con la migración proveniente de los países capitalistas subdesarrollados.

El propósito de este artículo es, precisamente, incursionar en esta problemática en un intento por dilucidar las causas, características e implicaciones de la migración en un ámbito que trasciende las relaciones sociales de producción propiamente capitalistas, pero que a la vez se vincula con éstas a través del intercambio internacional. Nos proponemos, en este sentido, desentrañar la naturaleza de la emigración cubana - yendo más allá del cliché explicativo que aduce la motivación política como esencial en ese procesoy aportar elementos de fondo que posibilitan dilucidar orgánicamente sus causas y limitaciones intrínsecas.

\section{SubdeSARRollo Productivo SOCIALISTA Y MIGRACIÓN INTERNACIONAL: ELEMENTOS TEÓRICO-CONCEPTUALES}

Cuba tiene una matriz organizativa y productiva distinta a la predominante en el contexto continental en el que se inserta. Su especificidad se sintetiza en el concepto de subdesarrollo productivo socialista. En este concepto, las nociones de subdesarrollo, desarrollo humano, heterogeneidad estructural e inestabilidad estructural ocupan un lugar central. Si bien teóricamente se elimina la relación capital/trabajo en la isla, en la práctica, las relaciones de producción derivadas del cambio en las formas de propiedad, de privada a colectiva, no dieron lugar a cambios que incentivaran la productividad. En el sistema de redistribución del ingreso, si bien mejoraron las condiciones de calificación y reproducción de la fuerza de trabajo, el salario, regido por un principio de homogeneización 
relativa y pleno empleo, ${ }^{2}$ lejos de incentivar el incremento de la producción y el desarrollo eficaz de las fuerzas productivas, tendió a desalentarlos. Esa situación inhibió la posibilidad de que en Cuba se establecieran mecanismos que estimularan la reducción de brechas socioproductivas y, por ende, que atacaran la problemática de la heterogeneidad estructural.

Aun cuando dicha política posibilitó cierto desarrollo de las fuerzas sociales de trabajo mediante procesos de tecnificación, especialización, profesionalización y educación, dicho desarrollo se hizo sobre una base dependiente, al amparo del intercambio internacional socialista (preferencial hasta 1989). Y aunque se trata de un debate inconcluso, en este fenómeno subyace una cierta subvaloración teórica de la ley del valor en el socialismo. Ello ocurre en virtud de que el valor de la fuerza de trabajo, teóricamente ajena a dinámicas de explotación, se disocia de los niveles de productividad. La retribución de la fuerza de trabajo - con una destreza media relativamente alta - no se rige por la contribución y capacidad de cada cual. Es así que, bajo un prisma ético de superación del antiguo sistema capitalista subdesarrollado, la percepción política de un sistema equitativo hizo que por décadas se mantuvieran ingresos relativamente igualitarios para el conjunto de la población trabajadora, lo que acabó por anular teórica y prácticamente los incentivos socialistas para el crecimiento de la productividad del trabajo. De aquí que el cambio en las relaciones sociales, en vez de propiciar transformaciones significativas en las dinámicas de generación de excedente económico, terminara por distorsionar los mecanismos de distribución social de la riqueza, así como sus alcances reales y potenciales.

Una reivindicación fundamental del régimen postrevolucionario $\mathrm{cu}-$ bano ha sido el impulso al desarrollo humano. ${ }^{3}$ Más allá de los encomiables

${ }^{12 /}$ Cabe destacar que esta medida resulta ajena al principio socialista de distribución del ingreso: «de cada cual según su capacidad, a cada cual según su trabajo» (Marx, 1974: 14) y se apega a una ley de población ajena a la capitalista, donde el ejército industrial de reserva desempeña un papel central (Marx, 1973, capítulo xxiII).

${ }^{13 /}$ Cabe destacar que Cuba sobresale en relación con los indicadores que propone el Pro- 
logros alcanzados en materia de equidad, educación y salud (Rushton, 2010), el derrotero del desarrollo en la isla ha estado marcado por los límites y las contradicciones que derivan del subdesarrollo productivo, los cuales se hacen más palpables a raíz del desmantelamiento de la Unión Soviética y la consecuente reinserción de Cuba al sistema capitalista mundial. Se generan en este contexto relaciones de intercambio desigual (Bernstein, 1982) que dan paso a la configuración de un círculo vicioso. ${ }^{4} \mathrm{Al}$ respecto, es preciso considerar que el excedente disponible para satisfacer formas diferenciadas de consumo o para cubrir inversiones es extremadamente limitado o prácticamente nulo cuando los niveles de productividad son relativamente bajos (Furtado, 1984). Y es precisamente esta limitante endógena la que explica la inestabilidad en torno a la sustentabilidad del desarrollo humano. Tómese en consideración que, en condiciones de baja productividad, la satisfacción de las necesidades fundamentales de la población reclama el empleo de una proporción elevada de la capacidad productiva. Más todavía, bajo un prisma de desarrollo humano, al acrecentarse dichas necesidades, tiende a producirse una creciente y a la postre insostenible inestabilidad en los procesos de redistribución, movilidad

grama de Naciones Unidas para el Desarrollo (PNUD, 1990) para medir el desarrollo humano. Empero, esos indicadores no evidencian fehacientemente las potencialidades para el mantenimiento del mismo, en cuanto a capacidades propias, articulación socioeconómica y relaciones sociales. A modo de ejemplo, las políticas sociales han permitido el logro de una condición desarrollada en el orden demográfico, con acceso gratuito a servicios de salud, educación y atención a grupos en desventaja; sin embargo, el deterioro paulatino en cuanto al logro de sus metas, que han ido experimentando esas políticas, se debe a la ausencia de un excedente capaz de utilizarse de manera proporcional en el gasto fiscal que demandan esas políticas, lo mismo que para cubrir las inversiones necesarias para su sostenimiento.

14/ Al respecto, Furtado (1993) afirma que el estancamiento (o círculo vicioso) que se mantiene en el subdesarrollo surge en relación a la interacción de las fuerzas que intervienen en el intercambio internacional y condicionan la formación de precios relativos. Estas fuerzas explican el proceso que influye en la pérdida del dinamismo económico 
social y equidad. A ello se agrega la carencia de encadenamientos eficaces que pudiesen servir como acicate para superar círculos viciosos en el orden productivo, sobre todo en espacios de interacción globales donde las desventajas del crecimiento endógeno se convierten en una traba de primera magnitud.

La compleja heterogeneidad estructural que caracteriza a la economía cubana tiene raíces históricas previas a la etapa revolucionaria, de raigambre colonial e imperialista. Las relaciones de producción establecidas a lo largo del periodo postrevolucionario no han permitido superar la condición subdesarrollada de la economía caribeña. Con todo, es preciso acotar que la inestabilidad estructural en la isla difiere, en esencia, del desequilibrio estructural presente en algunas sociedades latinoamericanas, entre otras cosas, debido a la ausencia de grandes corporaciones multinacionales, así como de unidades y agentes cuyo móvil es la maximización de la ganancia y la superexplotación laboral. Sin desconocer las marcadas diferencias en uno y otro contexto, el deterioro y el desaprovechamiento de las potencialidades de la fuerza de trabajo isleña son un hecho innegable. Esta situación emana de la limitada capacidad de acumulación que caracteriza al contexto cubano, la cual resulta no sólo insuficiente para cubrir las inversiones necesarias para sustentar dinámicas de crecimiento endógeno, sino para traducir ese crecimiento en mayores niveles de bienestar, consumo e ingreso para la población. En esta sui generis inestabilidad estructural, queda sucesivamente rezagada la productividad y los ingresos directos de la fuerza de trabajo, lo que redunda en una marginación progresiva del grueso de la población. A diferencia de otras sociedades, el afán de ganancia de las corporaciones no es lo que limita la ampliación del excedente socialmente disponible (CEPAL, 2010b), sino las distorsiones estructurales ${ }^{5}$ a las que hemos venido haciendo referencia.

en las sociedades latinoamericanas. En el caso cubano esta situación es agravada por el severo bloqueo impuesto a la isla por Estados Unidos y sus más acérrimos aliados.

${ }^{15 /}$ Este término es utilizado en el ámbito académico cubano para hacer referencia a los desequilibrios de la estructura productiva (Triana, 2008 y 2009; Torres, 2011). 
A partir de tales distorsiones, que el derrumbe del llamado socialismo real ha hecho más palpables, es posible explicar las condiciones estructurales y subjetivas que configuran y moldean los flujos migratorios contemporáneos en la isla, donde la limitación relativa del consumo y los exiguos mecanismos de movilidad social son parcialmente compensados a través de los flujos migratorios. Bajo estas circunstancias, se generan dos modalidades migratorias: a) la llamada migración económica, referida a la salida creciente de población en edad laboral que busca mecanismos de movilidad social allende las fronteras, y b) la denominada exportación de servicios intensivos, que alude al intercambio de fuerza de trabajo calificada por divisas. Ambas modalidades migratorias intentan mitigar - que no resolver - las limitaciones estructurales.

Para profundizar en la particular relación entre desarrollo y migración que caracteriza al contexto cubano es menester tener presente la noción de limitación relativa del consumo, la cual alude a la presencia de ingresos bajos, con reducidos diferenciales salariales, para una fuerza de trabajo con una relativamente alta capacitación. Teniendo en cuenta esta característica, lo que ha ocurrido con el proceso de reinserción de Cuba a las dinámicas internacionales de comercio y finanzas en las últimas décadas es que la desventaja comparativa de la isla en el mercado internacional ha redundado en una modalidad de intercambio desigual que ha afectado particularmente a la fuerza de trabajo. El deterioro paulatino de ésta última se manifiesta en virtud de que sus ingresos directos no logran cubrir la transferencia al consumo familiar o doméstico de los precios de las mercancías internacionales (más allá de una limitada canasta de productos de consumo básico) a través de las importaciones. Al no ser capaz el sistema productivo cubano de sustituir las importaciones o cubrir la demanda de la población, se ve compelida a recurrir, sin un excedente suficiente, a importaciones con precios que superan el ingreso medio de la población, incrementando el costo de la vida. Ello es posible por el exceso de circulante que se deriva tanto de las remesas y sus derramas para grupos sociales minoritarios, como por la venta de servicios que involucra a un segmento de la población económicamente activa con el concurso de Estado. De esa manera, 
se sufragan no sólo necesidades de los grupos sociales directamente involucrados, sino parcialmente también los costos de la redistribución socialista, aunque en ese proceso influyen otras dinámicas que favorecen la captación de divisas, como el turismo y el intercambio reciente con países aliados.

Si bien bajo la égida neoliberal los mercados laborales en los países capitalistas, y particularmente en los países capitalistas subdesarrollados o periféricos, se precarizan a grados extremos (Delgado Wise, 2013), en el caso de Cuba el deterioro de la fuerza de trabajo es resultado de la discordancia entre las capacidades de la fuerza de trabajo y la posibilidad de su aprovechamiento endógeno y liberalización relativa. Un aspecto visible de ese círculo vicioso es el subempleo que caracteriza a la isla. Cabe acotar, sin embargo, que esta situación comienza a experimentar cambios significativos a últimas fechas: de la política de pleno empleo sustentada por el Estado cubano se está transitando a mecanismos de incentivación, autoempleo y cooperativismo. Al abrirse nuevas opciones laborales y promoverse espacios de competitividad, la composición del empleo en la isla tiende a heterogeneizarse para dar lugar, aun sea de manera incipiente, a la emergencia de un mercado laboral.

En relación con esto último, es necesario señalar que algunas de las contradicciones manifiestas en las estrategias de desarrollo cubano se definen no sólo por la tentativa de evitar una confrontación abierta a través de las relaciones de intercambio entre diferenciales de productividad con el campo capitalista, sino también por el hecho de haber creado esquemas de valor propios y vínculos en la economía ajenos a la innovación tecnológica, la competitividad y el incremento del valor agregado a los bienes exportables. El círculo vicioso, que supuso encadenamientos productivos vinculados a transferencias internacionales basadas en el intercambio preferencial socialista, hizo que - como apuntamos antes- la relación entre productividad e ingresos de la fuerza de trabajo no se estableciera conforme a los estándares internacionales. Por el contrario, la fuerza de trabajo quedó circunscrita a un inestable proceso de retribución instaurado por medio de las políticas sociales y públicas, donde el pleno empleo devino en un logro moral desarticulado 
del principio socialista de distribución de acuerdo a los aportes y capacidades de cada individuo. De esta manera, la fuerza de trabajo registra actualmente un deterioro visible en sus ingresos que afecta sobremanera sus mecanismos de reproducción y movilidad social. Es por ello que paulatinamente la migración tiende a asumir un rol compensatorio, al fungir como factor contrarrestante de algunos de los efectos socioestructurales de las distorsiones propias del sistema productivo cubano y su interacción en el contexto global.

Haciendo abstracción del impacto del bloqueo estadounidense impuesto a la isla, las distorsiones estructurales internas no se explican por factores de dominación económica y política externa, como ocurre en el caso de la migración forzada bajo la égida del capitalismo neoliberal. ${ }^{6}$ En este sentido, más que un aislamiento del contexto global, se trata de una forma diferente de insertarse en él, donde las distorsiones estructurales son resultado del limitado uso endógeno de la fuerza de trabajo y su parcial compensación a través de la emigración. Este fenómeno se manifiesta como una secuela de los efectos de la heterogeneidad en el sistema de relaciones sociales internas con brechas económicas o sociales abiertas, tanto en el ámbito de las decisiones familiares e individuales relacionadas con la reproducción social, como en relación con los mecanismos de integración de la unidad nacional a los espacios de interacción globales.

Bajo la categoría compensación de distorsiones denotamos asimismo el flujo entre unidades estructurales diferenciadas, atendiendo a las dinámi-

${ }^{16 /}$ La noción de migración forzada se refiere a que, en el marco de la globalización neoliberal, «[...] las migraciones adquieren un nuevo papel dentro de la división del trabajo. Los mecanismos del desarrollo desigual generan condiciones estructurales, como el desempleo y las desigualdades, que catapultan las migraciones masivas de conjuntos poblacionales despojados y excluidos. Compelidos por la necesidad de acceder a medios de subsistencia u oportunidades de movilidad social, vastos sectores de la población son literalmente expulsados de sus territorios para desplazarse dentro del propio país o hacia el extranjero» (Márquez y Delgado Wise, 2011: 20).

1644 MIGRACIÓN Y DESARROLLO, VOL. 11, NÚM. 20, PRIMER SEMESTRE DE 2013 
cas de movilidad social y consumo, que en el caso de Cuba tienen que ver con los procesos de segmentación socio/clasista; procesos que aluden a las diferencias sociales derivadas de las disparidades en los ingresos, sin que éstas estén definidas por la posición del trabajador respecto de los medios de producción. Utilizamos también esta noción en alusión a la perentoriedad de incorporar fuerza de trabajo al comercio internacional como recurso para generar saldos positivos en la balanza comercial.

De esa manera, se entiende que la emigración cubana confluye en la propensión que se manifiesta en los flujos poblacionales a insertar fuerza de trabajo en las redes globales de producción, ${ }^{7}$ aportando, por esta vía, divisas para el sostenimiento de la estrategia de «desarrollo socialista» prevaleciente. Se genera así una tendencia a la ampliación del patrón migratorio con un sentido sistémico, en tanto factor de compensación frente a la crónica tendencia negativa que acusa la balanza comercial. La erosión paulatina del bienestar logrado en otras épocas incide en el mismo fenómeno, explicando la paradójica contradicción que entraña el hecho de que emigren personas con un estado relativo de bienestar que, desde un prisma de desarrollo humano, tendrían asegurada social e institucionalmente la subsistencia.

\section{INGRESOS Y DESIGUALDAD SOCIAL EN}

\section{LA TRAMA MIGRATORIA RECIENTE}

Para explicar el fenómeno migratorio cubano es necesario adentrarse en el andamiaje económico-social que impera en la isla, donde los ingresos y los salarios desempeñan un papel crucial. Se trata, empero, de variables depen-

${ }^{17 /}$ En el caso cubano dicha inserción se produce bajo una modalidad diferente de la reinserción de las economías periféricas a la esfera del capital monopolista internacional. En este último caso, las redes globales aluden al «[...] establecimiento de cadenas de 
dientes que condicionan la situación de pobreza de amplios sectores de la población (Vidal, 2008), ${ }^{8}$ donde, como se desprende de lo planteado en el apartado anterior, la baja productividad funge como un factor determinante.

Debido a que los ingresos no se constituyen en incentivos reales para el avance productivo, existe una clara disociación entre los niveles de productividad y los salarios e ingresos de la población. A esto se agrega una diferencia ostensible entre el salario nominal y el salario real derivada de una política estatal esencialmente restrictiva (Vidal, 2009). Algunos sectores presentan mayor capacidad para sostenerse conforme a sus ingresos, independientemente de las retribuciones indirectas contenidas en las políticas públicas. Con todo, un aspecto típico del empleo en el sistema productivo cubano es que está mayormente vinculado con el sector estatal, donde el salario es una variable independiente o exógena disociada del nivel de ocupación o producción. «Las empresas no deciden el nivel de los salarios sino que [éstos] son determinados centralmente por el Estado» (Vidal y Pons, 2008: 92). En este sentido, existe una correlación entre el incremento del gasto público y la designación de subsidios al empleo vía salarios, mientras que la demanda de bienes de consumo de la población posee cierta autonomía de la actividad empresarial (Vidal y Pons, 2008). Otro problema que enfrenta la medición de los ingresos es que las coberturas universales de salud y educación como ingresos para toda la población han significado un gasto desproporcionado, que no es contabili-

subcontratación y/o comercio intra-firma de las grandes corporaciones que se extienden como tentáculos al grueso de la geografía mundial. Dicha forma de expansión está dirigida a la reinserción de los países periféricos que disponen de recursos naturales y humanos abundantes y baratos, donde las nuevas plataformas de exportación operan bajo la modalidad de enclaves» (Márquez y Delgado Wise, 2011a: 7).

${ }^{18 /}$ Cabe apuntar que diversos autores cubanos han acuñado diversas categorías analíticas como las de «población vulnerable»y «pobreza con amparo» referidas por Espina Prieto (2009 y 2009a) o el concepto de "población en riesgo» propuesto por Ángela Ferriol et al. (2006) para dar cuenta del progresivo deterioro de la fuerza de trabajo. 
zado en la magnitud ostentada por los salarios reales. Aun así, es difícil para las familias cuyas entradas descansan en salarios y subsidios estatales efectuar gastos que vayan más allá de la alimentación (Togores y García, 2008).

De esa manera, un aspecto clave para comprender el deterioro palpable de la fuerza de trabajo es la relativa homogeneización de los ingresos. Aun cuando han existido estrategias para compensar ese quiebre, como el aumento a los salarios registrado en los últimos años y más recientemente la autocontratación y el autoempleo, ninguno de ellos se ha convertido todavía en un incentivo importante para incrementar la productividad y estimular la innovación. Con todo, estos cambios están conduciendo a una paulatina transformación de los agentes que participan en los procesos productivos, generando con ello una incipiente diferenciación social.

En esta vertiente, Mayra Paula Espina Prieto (2009a) advierte una re-estratificación social (cambios en la estructura social) caracterizada por el ensanchamiento de las estructuras económicas. Y si bien esta estratificación no guarda relación alguna con las condiciones de explotación basadas en la propiedad privada, constituye una respuesta fortuita a necesidades emanadas del carácter periférico del socialismo cubano, cuyos efectos más visibles han sido la ampliación de la pobreza como problema social y la expansión de la franja de población en situación de vulnerabilidad (Espina Prieto, 2009a). Se trata de un proceso que da paso a una determinada recomposición de la pequeña burguesía urbana y rural, por medio de la economía informal, el autoempleo y la ampliación de los mecanismos de mercado en la distribución social del ingreso. La mayor parte de los mecanismos de redistribución introducidos para administrar la crisis se concentra en la esfera de la circulación, por lo que se hace necesario dotar a la política social de una base de sustentabilidad económica endógena (Espina Prieto, 2009a). ${ }^{9}$ La liberación

19/ Esto implica encontrar estructuras y mecanismos de coordinación acordes a los procesos de complejización (heterogeneización social creciente, multiplicación de agentes

MIGRACIÓN Y DESARROLLO, VOL. 11, NÚM. 20, PRIMER SEMESTRE DE 2013 \67 
de los precios y la adecuación del consumo a la oferta y la demanda tienden a incrementar los niveles de productividad, aunque por tratarse de aspectos de una política reactiva que no ataca de raíz la problemática del subdesarrollo productivo socialista referida en el apartado anterior, contribuyen también, en el otro extremo, a la apertura de brechas sociales.

Bajo las circunstancias descritas, la emigración se convierte en una pieza importante del entramado descrito. A nivel macro, la exportación y la venta de servicios contribuyen a proveer divisas para el funcionamiento económico, aportando al mismo tiempo una válvula de escape para la fuerza de trabajo que no puede ser eficientemente empleada en la isla. A nivel micro, el mismo fenómeno explica las alternativas que se originan como resultado de la vulnerabilidad de algunos grupos sociales. Esta doble funcionalidad de la emigración cubana cuestiona las vertientes de interpretación que la visualizan, reduccionista e ideológicamente, como una actividad de índole esencialmente política relacionada con las exigencias y críticas del país que tradicionalmente ha figurado como su principal destino: Estados Unidos.

Para una exégesis sistémica es necesario comprender que la limitación relativa del consumo de la fuerza de trabajo propicia su deterioro paulatino y, para contrarrestarlo, la emigración es percibida falazmente como la única opción para un segmento amplio de la población económicamente activa. La compensación de distorsiones estructurales, por ello, le otorga valor dentro

económicos y actores sociales, de las interacciones entre ellos y de sus espectros posibles de acción, aumento de la intervención del azar, la incertidumbre y las cualidades emergentes, innovadoras en los cursos del futuro, entrelazamientos locales, nacionales y globales múltiples) que están teniendo lugar en los escenarios internos y el entorno internacional. Implica asimismo una reconstrucción de la política de igualdad: acciones que promuevan equidad y justicia social, corrijan desigualdades injustas, asuman las diferencias necesarias, aseguren retribuciones en el vínculo más estrecho posible con el aporte económico-social y protejan a los individuos y grupos que se encuentran en situaciones más precarias (Espina Prieto, 2009). 
de esa interpretación a la relación que desde la inestabilidad estructural se genera hacia el ámbito global (aspectos internos de desenvolvimiento económico y transformación social reflejados en el sistema de relaciones vigente). Este andamiaje teórico y conceptual reconoce la presencia de estrategias de movilidad social individual y familiar que, en un plano macroeconómico, buscan compensar distorsiones estructurales que, más que promover un «círculo virtuoso», permanecen atrapados en el «círculo vicioso», propio del subdesarrollo productivo que impera en la isla.

Los aspectos antes mencionados intentan complejizar el fenómeno de la emigración cubana no sólo como un traslado de personas, objetando las visiones que entienden ese proceso como oportunidades de desarrollo para las sociedades de origen y problemas sociales para las de destino. En cambio, la visión de la emigración cubana que hemos venido postulando reconoce rasgos de la heterogeneidad en la isla que propician la emigración, en un entorno socioeconómico, donde un sesgo importante lo va a constituir la «exportación» de niveles de conocimientos - por el peso que tienen en la balanza comercial— y la transferencia o «exportación» de fuerza de trabajo, incluyendo bonos demográficos, hacia otras latitudes. Se trata, en el fondo, de «aportes» o traspasos netos de la sociedad de origen a las sociedades receptora ${ }^{10}$ que, en el mediano y largo plazos, se traducen en una transferencia de excedentes y en una pérdida potencial de recursos y capacidades para el desarrollo de la isla.

Lo cierto es que Cuba presenta un saldo migratorio negativo progresivo que afecta sobremanera la composición sociodemográfica de la población. En una población de poco más de 11 millones de habitantes y que atraviesa por una fase avanzada de su transición demográfica, la emigración afecta el

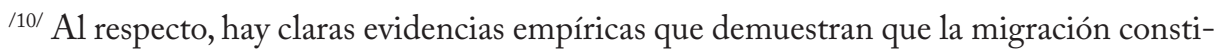
tuye en escancia un subsidio Sur-Norte y, por tanto, es apenas una salida coyuntural que a la postre refuerza los mecanismo de intercambio desigual que permean en el horizonte Norte-Sur (Delgado Wise y Márquez, 2012). 
crecimiento demográfico natural de la isla (Albizu Campos, 2009; Herrera y Hernández Castellón, 2009). Con todo, el recurso a la emigración se mantiene como una opción - aun sea de corto plazo — para compensar la inestabilidad estructural que caracteriza al subdesarrollo productivo cubano. Es por ello que a pesar de los relativamente elevados índices de desarrollo humano en la isla, no resulte incongruente, aunque sí paradójico, que emerjan nuevos destinos de la emigración cubana hacia países con índices de desarrollo humano menores a los registrados por la mayor de las Antillas. Otra paradoja asociada con la migración y el desarrollo humano en Cuba es que aquélla no sólo compensa déficits de consumo y oportunidades de movilidad social, sino que amplía y reproduce desigualdades sociales. Tómese en consideración que los migrantes son personas en edad laboral, con formación profesional en un grado elevado, cada vez más mujeres en edad reproductiva y blancos en su mayoría. Paulatinamente la emigración, como vía de movilidad social y como fuente de transferencias culturales y económicas, implica un proceso de transformación de la sociedad y del sistema de relaciones sociales. A través de ella, se crean vínculos extraterritoriales al nivel de la familia, no suficientemente asimilados por las instituciones en el país y que tienden a erosionar niveles supuestamente superados de equidad en términos de ingresos, raza y género; realidad que ha sido relativamente poco analizada en el ámbito de las ciencias sociales en Cuba.

$\mathrm{Y}$ es que los cambios en la reproducción social y el fracaso relativo de las políticas sociales orientadas hacia el aumento ininterrumpido de la «homogeneidad social» han solidificado una dinámica de transformación y reacomodo de los grupos sociales en intensa interrelación con lo que Mayra Paula Espina, Viviana Togores, Lilia Núñez, Lucy Martin y Gisela Ángel (2010) denominan elementos de «naturaleza externa, materiales, simbólicos y culturales» y que suponen una transformación de los actores en las distintas dimensiones de actuación, ${ }^{11}$ ya sea local, regional o nacional. En sentido es-

/11/ Trabajo, ingresos, salario, pobreza, vulnerabilidad, raza, género, medio ambiente.

1704 MIGRACIÓN Y DESARROLLO, VOL. 11, NÚM. 20, PRIMER SEMESTRE DE 2013 
tricto, el análisis referido supone un reconocimiento de características sociales en Cuba que desentonan con la connotación del concepto de desarrollo humano propuesto por el PNUD (1990), al admitir la presencia de grupos sociales en desventaja a pesar de los esfuerzos del Estado para el mantenimiento de la equidad y los derechos ciudadanos universales. En efecto, la emergencia de relaciones de inequidad revela que las políticas sociales sólo han cumplido parcial e insuficientemente su papel en la satisfacción de las necesidades de la población.

Como una secuela del fenómeno descrito, una paradoja más del desarrollo humano en la isla es la emergencia espontánea e institucionalmente reconocida de nuevas clases sociales con una recomposición de las capas medias, lo que da lugar a la emergencia de nuevos actores — asociados al autoempleo, las pequeñas y medianas empresas (Pymes), y la autogestión-, como es el caso de propietarios, patronos y empleadores que Mayra Paula Espina y colaboradores (2010) definen como pequeña burguesía urbana. Simultáneamente, se produce un proceso de segmentación al interior de las clases tradicionales en el socialismo (obreros, campesinos, dirigentes, directivos, empleados) $)^{12}$ propiciada por los vínculos con el sector emergente de la economía, así como con los nuevos sistemas de propiedad implementados en el sector agropecuario, donde la emigración, a través de las remesas, cumple un papel cada vez más relevante.

Más aún, Pavel Vidal (2008) hace referencia a que los grupos ajenos a los nuevos sistemas de propiedad, a los sectores emergentes y a las remesas, casi siempre salario-dependientes o empleados en el sector estatal en el área de los servicios, han sido los más afectados por el proceso de segmentación hacia lo interno de las clases sociales. Y una proporción importante de los sectores afectados son, precisamente, los profesionales. Dentro de estos últimos, por su

${ }^{112 /}$ Así se asumen por las ciencias sociales cubanas (clasificación asumida bajo el principio de que la clase política se distancia de las clases que representa). 
peso relativo en cuanto a la composición de género y por ser mayoría respecto de los hombres en la formación universitaria, sobresalen las mujeres.

La reinserción internacional actual ha traído aparejada la introducción relativa de relaciones monetario-mercantiles en el socialismo, como medidas un tanto desesperadas y hasta cierto punto incoherentes, para promover la eficiencia económica y la productividad. Según José Luis Rodríguez (2011), el aislamiento económico del trabajo individual y de los intereses personales y grupales frente a los sociales, obligó a utilizar categorías mercantiles para dotar de una nueva «racionalidad» a la división social del trabajo, aun cuando continúe predominando en Cuba la propiedad social sobre los medios de producción (Rodríguez, 2011). A modo de ejemplo, se podrían citar la racionalización del personal superfluo (así se le llama en los medios oficiales) en los centros laborales que implica una salida sustancial de las «almas» (utilizando otro vocablo cubano) empleadas por el Estado hacia la economía informal y la eliminación gradual de los subsidios a la alimentación, reorientándose la satisfacción de esta última progresivamente hacia los mercados de productos no racionados con mayores precios (Togores, 1996; Marquetti, 2002). Desde la creación de las tiendas en divisas ha ocurrido ese traspaso paulatino, sin el aumento correspondiente de los salarios, con la pretensión de controlar la inflación (Vidal, 2008).

Por ello, los ingresos salariales y de la seguridad social muestran una incapacidad para garantizar el acceso al consumo de bienes y servicios básicos (Espina et. al., 2010; Vidal, 2008), a pesar de sus incrementos sostenidos. En un grado no desdeñable, la dualidad monetaria (Triana Cordoví, 2009) supone «modelos de afirmación social» asociados a los ingresos en la moneda de mayor poder adquisitivo y sucedánea de las divisas internacionales. De aquí que las remesas se hayan convertido en un mecanismo sine qua non de movilidad social que da lugar a nuevos métodos y maneras de socialización.

Todo lo anterior plantea la necesidad de un cambio en el tratamiento «tradicional» del Estado hacia las comunidades de cubanos en el exterior, influida por el crecimiento de los asentamientos y los flujos de personas allende 
las fronteras nacionales. Este nuevo escenario está dando lugar al planteamiento de reformas, aún inconclusas, en materia de legislación migratoria, al tiempo que sugiere un perentorio cambio en las normas aplicadas sobre ciudadanía, derechos de los emigrantes y atención a la relativa pérdida que supone la migración en términos de costos sociales y educativos. En esta perspectiva, el Estado cubano anunció una serie de medidas de distensión con respecto a su comunidad en el exterior que entrarán en vigor a principios de 2013, las cuales, entre otras cosas, abaratarán los costos de trámites de salida y retorno, así como ampliarán posibilidades de repatriación. Con todo, la problemática de migración y desarrollo que enfrenta la isla reclama una visión de conjunto, que ataque sus raíces más profundas, con una visión de largo plazo que contemple la superación del subdesarrollo con una óptica antiimperialista y socialista.

\section{El MiTO DE LA MIGRACIÓN O}

\section{LA REALIDAD DEVELADA}

Paralelamente al crecimiento de los conflictos en el desarrollo económico, la introducción del turismo y el ensanchamiento de la crisis a inicios de la década de los noventa del siglo pasado, ocurre un desplazamiento del principal destino de la emigración cubana de Estados Unidos hacia otras latitudes. Al mismo tiempo, emergen nuevas modalidades de emigración que hoy se han consolidado en las prácticas y vías usadas por los emigrantes cubanos, tales como la del matrimonio con extranjeros, los contratos de trabajo y el cambio de ciudadanía. Algunas de las regiones con mayores asentamientos de cubanos tras el derrumbe de la Unión Soviética en 1989 son, en Europa, Alemania, Francia, España ${ }^{13}$ e Italia, y en América, además de Estados Uni-

13/ En el caso de España ocurre un ensanchamiento de las comunidades creadas en décadas anteriores que usan los recursos culturales y las redes creadas. Tiene un especial 
dos, México, Canadá y Costa Rica. En términos generales, la presencia de cubanos se ha extendido a casi todos los continentes.

La emigración reciente adquiere características similares, en su composición, al éxodo hacia Estados Unidos: se integra mayoritariamente por personas de piel blanca, acusa una firme tendencia a la feminización y abarca un significativo monto de profesionales. La motivación económica figura como la principal causa de la decisión de emigrar, observándose una clara correspondencia entre los apremios derivados de las carencias económicas y la decisión de emigrar. Por lo relativamente nóveles de estos flujos, la mayoría de los desplazamientos ocurre sin el soporte de redes sociales o experiencias familiares previas. Una cuestión que llama la atención al analizar los nuevos destinos es que los diferentes lugares de asentamiento guardan relación con los principales países emisores de turistas hacia Cuba, lo que coincide con la decisión del Estado de ubicar esta actividad como prioritaria en su estrategia de crecimiento económico. Hay una clara correspondencia, en este sentido, en el hecho de que países como Italia, Inglaterra, Alemania, Francia, Bélgica, Holanda y Suiza (en Europa), México, Brasil, Chile y Argentina (en América) se hayan constituido en destinos de la emigración cubana.

Por su parte, las remesas, según Manuel Orozco (2004, 2009), se canalizan principalmente a través del sector informal debido a que la mayoría de los cubanos prefieren realizar sus envíos por medio de mecanismos no oficiales. En torno al envío/recepción de remesas han proliferado sectores de empresarios cubanos en el exterior que controlan y dirigen estas operaciones empleando a terceros. Se trata de una práctica que se ha venido incrementando con los años y que asume modalidades diversas, condicionadas, en su mayoría, por los altos costos de las transferencias y las restricciones para su envío impuestas, en diversos periodos, por el gobierno estadounidense (Orozco, 2004).

peso la reunificación familiar y el traspaso de la ciudadanía a familiares con grados de consanguinidad cercanos. 
La entrada de remesas a Cuba ha tenido momentos de crecimiento y disminución. Lorena Barberia (2007) describe que antes de la crisis económica su entrada era esporádica y limitada por el conflicto con Estados Unidos, circunscribiéndose al envío de «paquetes» con ropa, alimentos y medicinas, fundamentalmente. Después de 1989 comienzan a desempeñar el papel de ingresos familiares, aceptándose socialmente como un mecanismo de acceso a espacios de consumo de bienes duraderos de uso cotidiano, sea para la alimentación o para otros fines (Fresneda, 2006). Y si bien en Cuba estos servicios son gratuitos y universales, resulta cada vez más evidente el papel desempeñado por la emigración como mecanismo de movilidad social y como factor compensatorio del deterioro en el ingreso, la capacidad de consumo y el bienestar social de la población cubana.

Cabe advertir que el término remesas es empleado en la isla para referirse a los recursos monetarios que ingresan al país, muchos de los cuales, en sentido estricto, no caben en la definición convencional de remesas: «[...] ingresos laborales enviados por emigrantes desde un país que no es el suyo a un familiar en su país de origen» (Orozco, 2005: 5). ${ }^{14}$ Bajo la acepción cubana, las remesas representan — según Méndez, Figueroa y Lloret (2006)— transferencias de rentas que pueden implicar o no una contraprestación en términos de bienes y servicio hacia o desde el resto del mundo. En el caso de las transferencias con contrapartida, éstas se refieren a los pagos netos de intereses de la deuda externa, remesas netas de utilidades, remesas de trabajadores cubanos en el exterior, primas e indemnizaciones por seguros u otras similares. Las transferencias sin contrapartida comprenden las donaciones materiales y financieras, aportes a organismos internacionales y pagos netos de impuestos y derechos consulares.

${ }^{114 /}$ Cabe apuntar que esta definición se ha ido sofisticando con el tiempo con fines operacionales. Entre otras cosas, el término emigrante ha sido reemplazado por el de residente y el de «remesas de trabajadores» por «transferencias personales» (Orozco, 2005: 8). 
La medición de las remesas por medio de las transferencias corrientes netas se dificulta por la no desagregación de datos en función de las donaciones recibidas, como también por el hecho de que las cifras oficiales se refieran a transacciones ajustadas a mecanismos legales, donde no se incluyen las cantidades relacionadas con el sector informal. Es por ello que existen estimaciones divergentes acerca del monto de las remesas captadas en Cuba. A modo de ejemplo, José Alejandro Trujillo (2001), cruzando variables sobre la población cubana residente en Estados Unidos y diversos rangos de envíos, estima el monto de remesas entre 300 y 700 millones de dólares. Por su parte, la Comisión Económica para América Latina y el Caribe (CEPAL), que en 1999 estimaba su monto en alrededor de 700 millones de dólares, en 2010 sostiene que las remesas han crecido a grado tal de convertirse en un elemento fundamental para dinamizar el mercado interno en el proceso de actualización del modelo económico cubano, precisando que, producto del relajamiento de las restricciones desde Estados Unidos, éstas ascienden al orden de dos mil millones de dólares (CEPAL, 2010a).

Frente a la distribución regresiva del ingreso por la que atraviesa actualmente la isla y la cancelación gradual del aseguramiento de la alimentación y los artículos de primera necesidad mediante las políticas de subsidios (Marquetti y García Álvarez, 1999), las remesas se han erigido en un pilar importante de la estrategia estatal para «[...] hacer frente al consumo social y a las inversiones requeridas por la economía» (Rodríguez, 2011: 33).

La exportación de servicios intensivos cumple un papel fundamental en esta última perspectiva. Se trata, en esencia, de la promoción institucional de la exportación de fuerza de trabajo calificada para la obtención de divisas. Esta opción migratoria está incluida en la más reciente gestión del Estado en pro de la actualización del modelo económico cubano. Más allá de la concepción inicial de esta política, como una oferta solidaria de servicios en el marco del internacionalismo socialista, por esta vía se pretenden utilizar los excedentes de fuerza de trabajo calificada que no pueden ser aprovechados internamente para compensar las distorsiones estructurales que acusa el aparato producti- 
vo. Asimismo, atendiendo a las expectativas de consumo y movilidad social de quienes participan en esta actividad, se promueve una migración de retorno o temporal modulada a través de las proyecciones de política exterior del Estado cubano.

Aun cuando la exportación de servicios intensivos ha cobrado una fuerte presencia en la actualidad, Nestor Marimón y Evelyn Martínez (2010) exponen que se trata, en realidad, de una práctica de vieja data que compromete sobre todo al sector de la salud y cuyos antecedentes se remontan a 1963, cuando un grupo de personal médico compuesto por 55 colaboradores se desplazó a Argelia. A lo largo de casi 50 años se ha diversificado la cooperación médica tanto en lo científico como en la docencia, con un total de colaboradores que asciende a 134 mil 849 distribuidos en 108 países del mundo. ${ }^{15}$ Sin embargo, paulatinamente la exportación de servicios intensivos se ha venido extendiendo a otras áreas, involucrando a profesionales de diferente formación, dirigida sobre todo a países de la periferia, por presentar rasgos comunes y ambientales, con analogías en las especializaciones agrícolas y agroalimentarias. En este ámbito, destacan naciones como Bolivia, Venezuela y Ecuador. Esta estrategia también se ha extendido a algunos países desarrollados como es el caso de Italia (ANSA, 2010).

La peculiar funcionalidad de la exportación de servicios intensivos para los profesionista involucrados estriba en que, aunque migran temporalmente, siguen siendo una importante fuerza laboral para la isla, al adquirir en el exterior otros ingresos y facilidades para obtener bienes para la familia. Se trata de un proceso masivo que de manera ostensible ha coadyuvado a que, paradójicamente, esos sectores experimenten procesos relativos de movilidad social y alcancen un cierto estatus asociado a su profesión.

115/ En África 38 países con 45 mil 488 colaboradores. En América, 39 países con 79 mil colaboradores, y en Euro Asia y el Medio Oriente, 31 países con 9 mil 644 colaboradores (Marimón y Martínez, 2010). 
Aprovechando estas características se crea, en la actualidad, una empresa comercializadora de servicios médicos, que no sólo exporta ese tipo de servicios sino que paulatinamente abrirá espacios para la atención médica a pacientes extranjeros en la isla. Esa estrategia contiene un paquete de más de 100 servicios médicos, donde resaltan la cirugía cardiovascular e intervencionista, la oftalmología, la ortopedia y la traumatología, la cirugía de mínimo acceso y general, la gastroenterología y los implantes dentales. Se incluyen también los servicios relacionados con la calidad de vida, la cirugía reconstructiva y la cosmética. El objetivo es hacer sostenible y eficiente el sistema nacional de salud (Cubadebate, 2011).

Esta vía para la compensación de distorsiones estructurales, lejos de ser exclusivamente un acto institucional consciente, adquiere una connotación pragmática en el legítimo e intangible vínculo que existe entre el insuficiente ingreso, el crecimiento del costo de la vida, las posibilidades de acceso a bienes duraderos para la familia y la vocación humanista de la medicina y educación cubanas. Al mismo tiempo, el hecho de que los servicios intensivos adquieran cada vez más un papel relevante en la contracción del déficit financiero y comercial que padece la isla de manera crónica, implica reconocer —en un sentido macroestructural- a ese tipo de intercambio como un proceso de migración temporal con consecuencias favorables para la familia en el acceso a consumo de alimentos, bienes duraderos, ropa, calzado, autos, entre muchos otros. Se trata de satisfacer expectativas de consumo y movilidad social similares a las que se manifiestan como motivación del acto de emigrar en las salidas definitivas, así como mediante contratos de trabajo en el exterior, matrimonios con extranjeros con anuencia familiar, entre otros (Fresneda, 2006).

Más allá de los alcances reales de la estrategia descrita, es claro que no está dirigida a modificar las causas de fondo de las distorsiones estructurales que caracterizan al subdesarrollo productivo socialista. Al intentar definirla como una emigración laboral, ha de tenerse en cuenta que, en este caso, la fuerza de trabajo se inserta al mercado laboral internacional a través de condiciones definidas por la sociedad de origen, sin una exposición a condicio- 
nes laborales deshumanizadas, o definidas por los países de destino. En este sentido, se trata de una estrategia institucional que contrasta radicalmente con los programas de trabajadores temporales promovidos por las principales potencias capitalistas bajo la égida de la globalización neoliberal. Se trata, además, de una estrategia de exportación de fuerza de trabajo que permite aprovechar, hasta cierto punto y con un limitado impacto en el desarrollo económico endógeno, el enorme potencial en recursos humanos calificados con que cuenta Cuba. Al mismo tiempo, ello posibilita compensar, aunque ineficazmente y de una manera coyuntural, las distorsiones que representan círculos viciosos en la economía.

Este proceso semeja, en sus alcances, a la migración económica definitiva dirigida a Estados Unidos, España y otros destinos, porque si bien genera ingresos familiares y abre hasta cierto punto canales de movilidad social al tiempo que genera divisas, profundiza los problemas de fondo en la heterogeneidad productiva cubana. Tómese en consideración que la exportación de fuerza de trabajo implica, en sí misma, una modalidad de intercambio desigual (Delgado Wise y Márquez, 2012), al implicar una transferencia y traslado de recursos humanos al exterior que crecieron y fueron educados en la isla y que potencialmente debieran ser aprovechados para el desarrollo endógeno. El problema se acentúa en virtud de las limitaciones demográficas de la isla. Sin adoptar una postura malthusiana, es preciso tomar en consideración que Cuba se encuentra en una fase avanzada de su transición demográfica y que la exportación de fuerza de trabajo a través de la migración tiene límites demográficos y graves consecuencias socioeconómicas para el desarrollo futuro.

\section{Conclusiones}

Las peculiaridades de la relación entre migración y desarrollo en Cuba están permeadas por las dinámicas de desarrollo desigual que emergen de la condi- 
ción subdesarrollada de la isla y su inserción en un contexto capitalista signado por la globalización neoliberal y el bloqueo impuesto por Estados Unidos y sus aliados. Las distorsiones estructurales que acompañan a este fenómeno tienen como telón de fondo el deterioro de la fuerza de trabajo expresado en una limitación relativa del consumo y en la ausencia de canales de movilidad social, que operan como elementos explicativos del flujo migratorio contemporáneo en el país antillano. Lo paradójico, en todo caso, es que en un ámbito caracterizado por el cierre de brechas sociales, sean restricciones de índole social las desencadenantes de los flujos migratorios. Más aún, es pertinente advertir que, aunque por causas diferentes, al interior de la isla comienzan a prefigurarse procesos que guardan relación con el contexto de desarrollo desigual y de distribución inequitativa de la riqueza que caracterizan al capitalismo contemporáneo. Y si bien Cuba pareciera estar disociada aún de las redes globales de capital monopolista inherentes a la globalización neoliberal, su fuerza de trabajo paulatinamente se va insertando tangencialmente en ellas, con previsibles impactos negativos para su desarrollo. No debe perderse de vista que la migración entraña una nueva modalidad de intercambio desigual para la isla, en virtud de que aspectos clave de la formación y reproducción de su fuerza de trabajo acaban por ser transferidos a otros contextos. ${ }^{16}$

Tácitamente, el intríngulis detrás del logro del desarrollo humano se expresa, por un lado, en las crecientes limitaciones en materia de productividad y generación de excedentes y, por el otro, en el papel que paulatinamente la fuerza de trabajo se ve compelida a desempeñar para compensar las distorsiones socioeconómicas que emergen de dichas limitaciones. No obstante, el subempleo, la inequidad, la necesidad de acceder a medios de subsistencia externos para compensar el ingreso familiar y la carencia de mecanismos de movilidad social son aspectos de la realidad social que paulatinamente se ubi-

${ }^{116 /}$ Tal es el caso de los costos educativos y de salud, y de la capacidad de innovación desaprovechada de su fuerza de trabajo altamente calificada.

1804 MIGRACIÓN Y DESARROLLO, VOL. 11, NÚM. 20, PRIMER SEMESTRE DE 2013 
can en la base de la migración isleña, sin que ello derive del sometimiento de la fuerza laboral cubana a relaciones internas de explotación. De aquí que resulte irónico que el caso cubano dé cuenta de un flujo migratorio de una nación no capitalista - que mantiene una condición subdesarrollada y dependiente- hacia destinos capitalistas, en el marco de la globalización neoliberal.

Por último, cabe destacar que con las recientes medidas migratorias, el flujo cubano pareciera encontrar espacios de canalización e identificación institucional, que resguardan, aun sea insuficientemente, los derechos de los migrantes y sus garantías al tomar la decisión de migrar. No puede desconocerse, sin embargo, que a pesar de la implementación de mecanismos proteccionistas en relación con la salida de algunos segmentos de la fuerza de trabajo altamente calificada, estas medidas acaban circunscribiéndose -quiérase o no- a la errada percepción de la emigración como una alternativa de desarrollo. Bajo esta óptica, la migración cubana, en tanto expresión de las contradicciones emanadas del subdesarrollo productivo socialista, se convierte en una opción para enfrentar, más no resolver, la particular heterogeneidad productiva y social que persiste en la isla y que se acentúa en el contexto de la crisis sistémica en curso. Reencauzar a Cuba por una senda de desarrollo socialista que ataque de raíz las causas y las secuelas de su subdesarrollo productivo, aprovechando sus elevados niveles de desarrollo humano, constituye el gran desafío para afrontar el peculiar nexo entre migración y desarrollo que distingue a la mayor de las Antillas hoy.

\section{Referencias}

Albizu-Campos Espiñeira, Juan Carlos (2009), «Dinámica demográfica», en Agencia Española de Cooperación Internacional para el Desarro1lo, Miradas a la economía cubana II, La Habana, Editorial Caminos. Ansa (Asociación de Naciones del Sudeste Asiático) (2010), «Acuerdo de Cooperación Cuba-Italia en cirugía médica», 〈http://www.5av.it/no- 
tizie/75-news-salute-medicina/1919-acuerdo-de-cooperacion-italia-cuba-en-cirugia-medica-.html> (consulta: noviembre de 2012).

Barberia, Lorena (2002), «Remittances to Cuba: An Evaluation of Cuban and us Government Policy Measures», Rosemarie Rogers working paper series, Working Paper, número 15.

Bernstein, Henry (1982), «Industrialization, Development and Dependence» en Hamza Alavi y Teodor Shanin (editores), Introduction to the sociology of developing societies, Reino Unido, MacMillan.

CePal (Comisión Económica para América Latina) (2010a), Cuba: Evolución económica durante 2010 y perspectivas para 2011, Chile, CEPAL.

(2010b), «Migración internacional, derechos humanos y desarrollo en América latina y el Caribe», «http://www.eclac.org/celade/〉 (consulta: noviembre de 2012).

Cubadebate (2011), «Presentarán empresa comercializadora de servicios médicos en FIHAV», «http:/www.cubadebate.cu/noticias/2011/11/01/ presentaran-empresa-comercializadora-de-servicios-medicos-en-fihav/s (consulta: noviembre de 2012).

Delgado Wise, Raúl (2013), «The migration and labour question today: new imperialism, unequal development and forced migration», Monthly $R e-$ vierw, volumen 65, número 2 .

, Humberto Márquez y Ruben Puentes (2013), «Reframing the debate in migration development and human rights», Population, space and place, volumen 19, número 4 (en prensa).

y Humberto Márquez (2012), «Strategic dimensions of Neoliberal Globalization: the Exporting of Labor Force and Unequal Exchange», Advances in Applied Sociology, volumen 2, número 2.

Espina Prieto, Mayra Paula (2009), Desarrollo, Desigualdad y politicas sociales. Acercamiento desde una perspectiva compleja, La Habana, Ediciones Acuario.

(2009a), Politicas de atención a la pobreza y la desigualdad, Buenos Aires, CLACSO. 
, Viviana Togores, Lilia Núñez, Lucy Martin y Gisela Ángel (2010), «Proyecto Heterogenización social y Política de Equidad», Talleres de Diálogo, Informe Sistematización de estudios sobre heterogeneidad social y desigualdades en Cuba, 2000-2008, La Habana, cIP.

Feinsilver, Julie (2006), «Cuban medical diplomacy: when the left has got it right», Foreign Affairs, número 6.

Ferriol, Ángela (2003), «Acercamiento al estudio de la pobreza en Cuba», Texas, LASA.

, Maribel Ramos y Lia Añé (2006), «Reforma económica y población en riesgo en Ciudad de la Habana», Revista Investigación Económica, INIE, números 1-2, enero-junio.

Foster, John Bllamy, Robert W. MacChesney y Jamil Jonna (2011), «The Global Reserve Army of Labor and the New Imperialism», Monthly Review, volumen 63, número 6.

FRESNEDA, Edel J. (2006), Vivir con lo que me mandan: las remesas y su impronta en la familia cubana, Buenos Aires, clacso.

Furtado, Celso (1984), Desarrollo y Subdesarrollo, Buenos Aires, eudeba.

(1993), Los vientos del cambio, México, Fondo de Cultura Económica.

Harver, David (2004), El nuevo imperialismo, Madrid, Akal.

Herrera, Lorenzo y Lorenzo Hernández Castellón (2009), «Crecimiento de la población», Cuba, población y desarrollo, La Habana, Centro de Estudios Demográficos de la Universidad de la Habana.

Marimón Torres, Nestor y Evelyn Martínez Cruz (2010), «Evolución de la colaboración médica cubana en 100 años del Ministerio de Salud Pública», Revista Cubana de Salud Pública, volumen 36, número 3.

Marquetti Nodarse, Hiram y Anicia García Álvarez (1999), Proceso de reanimación del sector industrial. Principales resultados y problemas, La Habana, Centro de Estudios de la Economía Cubana. (2002), La coyuntura financiera de Cuba al culminar el 2002, La Habana, Centro de Estudios de la Economía Cubana.

Márquez, Humberto y Raúl Delgado Wise (2011a), «Una perspectiva del 
sur sobre capital global, migración forzada y desarrollo alternativo», Migración y Desarrollo, volumen 9, número 16.

(2011b) «Claves analíticas de la migración Mexicana a Estados Unidos», Observatorio del Desarrollo, volumen 1, número 1.

Marx, Carlos (1973), El Capital (tomo I), La Habana, Ciencias Sociales.

(1974) «Crítica del programa de Gotha» en Carlos Marx y Federico Engels, Obras escogidas, Moscú, Editorial Progreso.

Méndez Delgado, Elier, María Elena Figueroa Gonzáles y María del Carmen Lloret Feijóo (2006), Antecedentes y actualidad de la medición macroeconómica, edición electrónica, 〈http://www.eumed.net/ libros/2006b/emd2/> (consulta: diciembre de 2012).

OIT (Organización Internacional del Trabajo) (2011), Tendencias mundiales del empleo 2011: el desafio de la recuperación del empleo, Ginebra, oIT.

Orozco, Manuel (2004), The Remittance Marketplace: Prices, Policies and Financial Institutions, Georgetown University, Washington, Institute for the Study of International Migration.

(2005), «Consideraciones conceptuales, retos empíricos y soluciones para la medición de remesas», Inter-American Dialogue, Informe presentado a la reunión del Centro de Estudios Monetarios Latinoamericanos (CEMLA), México, 〈http://www.cemla-remesas.org/medicion/PDF/ seminariomx/O5 IOMX-ES-DOC-Descriptivo.pdf) (consulta: diciembre de 2012).

(2009), «The Cuban Condition: Migration, Remittances, and its Diaspora», Inter-American Dialogue, conferencia: "El Caribe en su Inserción Internacional», sponsored by the cIPR/ciAPA, San José, Costa Rica, febrero 3 y 4.

PNUd (Programa de Naciones Unidas para el Desarrollo) (1990), Informe de Desarrollo humano, Bogotá, Tercer Mundo Editores.

Rodríguez, José Luis (2011), Notas sobre la economía cubana, La Habana, Ruth Casa Editorial.

Rushton, Marc (2010), Socialist Humanism and the Cuban Revolution, tesis 
para la obtención del grado de Doctor en Estudios del Desarrollo, Universidad Autónoma de Zacatecas, Unidad Académica en Estudios del Desarrollo.

SÁnchez VÁzquez, Adolfo (1999), Entre la realidad y la utopia: ensayos sobre política, moral y socialismo, México, Fondo de Cultura Económica.

Togores González, Viviana (1996), «Enfoque social del desempeño de la economía cubana en 1996», La economía cubana en 1996: resultados, problemas y perspectivas, La Habana, Centro de Estudios de la Economía Cubana.

y García Anicia (2008), Consumo, mercados y dualidad monetaria en Cuba, La Habana, Centro de Estudios de la Economía Cubana.

Torres Pérez, Ricardo (2011), «La actualización del modelo económico cubano: continuidad y ruptura», Temas, 8 de junio.

Triana Cordoví, Juan (2008), De los desequilibrios a las distorsiones. Cómo crecer en el futuro Inmediato, La Habana, Centro de Estudios de la Economía Cubana.

(2009), Economía basada en el conocimiento, La Habana, Centro de Estudios de la Economía Cubana.

Trujillo Aguilar, José Alejandro (2001), «Las remesas desde el exterior: Un enfoque metodológico analítico», Cuba Investigación Económica (La Habana), número 3, julio-septiembre.

Vidal Alejandro, Pavel (2008), Determinantes de la inflación en Cuba: una modelación estructural, La Habana, Centro de Estudios de la Economía Cubana.

(2009), El salario real y la productividad en Cuba: actualización, La Habana, Centro de Estudios de la Economía Cubana.

y Saira Pons (2008), Determinantes de la inflación en Cuba: una modelación estructural, La Habana, Centro de Estudios de la Economía Cubana. 